\title{
DEFORMATION OF OPEN EMBEDDINGS OF $Q$-MANIFOLDS
}

\author{
BY
}

\author{
A. FATHI AND Y. M. VISETTI
}

\begin{abstract}
We prove here for Hilbert cube manifolds the full analogue of the Cernavskii-Edwards-Kirby Theorem concerning the deformation Principle for open embeddings of topological manifolds.
\end{abstract}

0. Introduction. In $\left[\mathbf{C}_{2}\right], T$. A. Chapman proved the local contractibility of the homeomorphism group of a $Q$-manifold. His recent simplified proof involves a certain "Handle Lemma" (see $\$ 1$ below) which does not lead directly to the full infinite-dimensional analogue of the Cernavskil-Edwards-Kirby Deformation Theorem, nor to its corollaries, whose proofs are very formal if derived from a good relative Deformation Principle, as explained in [EK] or [S]. We show here how to derive a "strong" Handle Lemma from Chapman's one, using a new "weak" Handle Lemma ( $\$ 1)$, and then we give some indications on the usual chart by chart induction to obtain this relative principle (\$2). As an amusing consequence, we prove that no $Q$-manifold supports any topological group structure (§3), a fact asserted by R. D. Anderson and N. Kroonenberg in their infinite-dimensional problems list $[\mathbf{A K}]$, presumably on the basis of a different proof. Among the general consequences of the Deformation Theorem, which appear in [EK] and [S], let us mention the extension of isotopies (defined in a neighborhood of a compactum), and the submersion theorem (see also [CK]): a proper submersion whose fibers are $Q$-manifolds is a locally trivial fiber bundle. For more corollaries, see $\S 3$.

We want to thank T. A. Chapman for explaining to us his new proof of local contractibility, R. D. Edwards for detecting our stupidities and for very helpful conversations, and L. C. Siebenmann for bringing to our attention this problem and for his helpful encouragements.

Recall that the Hilbert cube $Q$ is the countable product of closed intervals (i.e. $Q=[0,1]^{\infty}$ ). A Hilbert cube manifold (or $Q$-manifold) is a separable metric space which has an open cover by sets (called "charts") homeomorphic

Received by the editors April 2, 1975.

AMS (MOS) subject classifications (1970). Primary 58B99; Secondary 57E05, 58n10.

Key words and phrases. $Q$-manifold, open embedding, isotopy, compact open topology, canonical deformation, local contractibility. 
to open subsets of $Q$. For the basic properties of $Q$-manifolds, in particular those directly related to Anderson's notion of $Z$-set, we refer to Chapman's notes on the subject $\left[\mathrm{C}_{3}\right]$.

Our goal is to prove the following:

Deformation Theorem. Let $M$ be a $Q$-manifold, $U \subset M$ be open, and $C \subset U$ be compact. Let $D \subset V$ be closed subsets of $M$, such that $V$ is a neighborhood of $D$. Then the following holds:

$D(M ; D, V, C ; U):$ if $h: U \rightarrow M$ is an open embedding equal to the identity inclusion $i: U \rightarrow M$ on $U \cap V$, and $h$ is sufficiently near to $i: U \rightarrow M$ (for the compact-open topology), then there exists an isotopy $h_{t}, 0 \leqslant t \leqslant 1$, of $h$ through open embeddings $h_{t}: U \rightarrow M$ such that $h_{1}=i$ on $D \cup C$ and $h_{t}=h$ on $D$ and outside some compact set in $U$ (independent of $t$ and even of $h$ ). Further the isotopy is canonical in the sense that it is a continuous function of $h$ (in the compact open topology) as $h$ varies sufficiently near $i$. Also $h_{t}=i$ in case $h$ $=i$

In fact, a $Q$-manifold has a basis of open sets which are (abstractly) open cones on compacta (more precisely $Q$ is homogeneous and homeomorphic to its own cone $c Q$, see $\left[\mathrm{C}_{3}\right]$ ). So that, as is explained in $\$ 0$ of $[\mathbf{S}]$, to find the isotopy between $h$ and $h_{1}$ is a simple application of Alexander isotopies, once we prove the following weaker statement:

$D^{\prime}(M ; D, V, C ; U):$ If $h: U \rightarrow M$ is an open embedding equal to the identity inclusion $i: U \rightarrow M$ on $V \cap U$, sufficiently near $i$ (for the compact open topology), then there exists an open embedding $h_{1}: U \rightarrow M$ such that $h_{1}=i$ on $D \cup C$ and $h_{1}=h$ outside some compact set in $U$ (independent of $h$ ). Further the assignment $h \rightarrow h_{1}$ is canonical in the sense that it is a continuous function of $h$ for the compact open topology, and $h_{1}$ is the identity if $h$ is.

1. Handle Lemma. Let $\mathbf{R}^{n}$ be the n-euclidian space with its standard metric, and $B^{n}$ (resp. $S^{n-1}$ ) its unit ball (resp. unit sphere). Denote by $d$ any metric on $B^{k} \times \mathrm{R}^{n} \times Q$, which is the standard metric on $B^{k} \times \mathrm{R}^{n}$ and a metric on $Q$. The $n$-torus $T^{n}$ is viewed, say, as the quotient space $R^{n} / 8 Z^{n}$.

In order to simplify the notations, we use the following conventions. Let $(k, n)$ be an arbitrary fixed pair of nonnegative integers. We put $H=B^{k} \times \mathrm{R}^{n}$; $H_{r}=B^{k} \times r B^{n}$ and $\partial H_{r}=S^{k-1} \times r B^{n}$ (any $r>0$ ). Double underlining indicates "stabilization" by $Q$; for example: $\underline{\underline{H}}_{r}=B^{k} \times r B^{n} \times Q=H_{r} \times Q$, more generally $\underline{\underline{X}}=X \times Q$ for any space $X$.

1.1. Chapman's Handle Lemma $\left[\mathrm{C}_{4}\right]$. Let $h: \underline{\underline{H}}_{3} \times[0,1] \rightarrow \underline{\underline{H}} \times$ $[0,1]$ be an open embedding sufficiently close to the identity, which has the 
following properties:

(*) $h \mid \partial H_{3} \times[0,1]$ is the identity,

(**) $h \mid \underline{\bar{H}}_{3} \times 0$ is the identity.

Then one can canonically associate to $h$ an open embedding $h_{1}: \underline{H}_{3} \times[0,1]$ $\rightarrow \underline{=} \times[0,1]$, with the properties (*), (**) and such that $h_{1}$ is the identity on $\underline{H}_{1} \times[0,1]$ and $h_{1}=h$ outside $\underline{H}_{2} \times[0,1]$. Moreover $h_{1}$ is the identity if $h$ is.

We will prove below the following complementary result:

1.2. WeAK Handle Lemma. Let $h: \underline{H}_{6} \times[0,1] \rightarrow \underline{\underline{H}} \times[0,1]$ be an open embedding, sufficiently close to the identity, with the following properties:

(*) $h \mid \partial \underline{H}_{6} \times[0,1]$ is the identity.

Then one can canonically associate to $h$ an open embedding $h_{1}$, with the property (*), such that $h_{1}=h$ outside $\underline{H}_{5} \times[0,1]$, and:

(**) $h_{1} \mid \underline{H}_{3} \times 0$ is the identity.

Moreover $h_{1}$ is the identity if $h$ is.

From 1.1 and 1.2, we deduce easily the following general version:

1.3. Strong Handle Lemma. Let $h: B^{k} \times 6 \dot{B}^{n} \times Q \rightarrow B^{k} \times \mathbf{R}^{n} \times Q b e$ an open embedding, sufficiently close to the identity with the property:

(*) $h \mid S^{k-1} \times 6 B^{n} \times Q$ is the identity.

Then one can canonically associate to $h$ an open embedding $h_{1}$ with the property (*) and such that:

(i) $h=h_{1}$ outside $B^{k} \times 5 \dot{B}^{n} \times Q$,

(ii) $h$ is the identity on $B^{k} \times B^{n} \times Q$. Moreover $h_{1}$ is the identity if $h$ is.

Proof of 1.3 From 1.1 AND 1.2. We view $Q$ as $Q \times[0,1]$ by singling out its first coordinate interval; then $h$ is an open embedding of $\underline{H}_{6} \times[0,1]$ into $\underline{\underline{H}} \times[0,1]$. By 1.2 , we can canonically arrange that $h$ becomes the identity on $\underline{\underline{H}}_{3} \times 0$, without changing it on $\underline{\underline{H}}_{6} \times[0,1]$ and outside $\underline{\underline{H}}_{5} \times[0,1]$. Denote by $g$ the resulting open embedding. Now $g$ restricted to $\underline{\underline{H}}_{3} \times[0,1]$ satisfies the hypotheses (*) and (**) of Chapman's Handle Lemma, which provides continuously for the compact open topology a straightened "handle" $h_{1}$. In particular $h_{1}=g$ outside $\underline{\underline{H}}_{2} \times[0,1]$, so that we can extend it, by means of $g$, to an open embedding of $\underline{H}_{6} \times[0,1]$ into $\underline{\underline{H}} \times[0,1]$, that we still denote by $h_{1}$. If we now reintegrate $[0,1]$ as the first coordinate interval of the Hilbert cube, we obtain an open embedding $h_{1}: B^{k} \times 6 \dot{B}^{n} \times Q \rightarrow B^{k} \times \mathbf{R}^{n} \times Q$ which has the required properties.

Proof of the Weak Handle Lemma 1.3. We construct the (weakly) straightened embedding $h_{1}$ in several steps, and it will be supposed implicitly at 
each stage that we work with "handles" so close to the identity that all the constructions are possible. We start with the given open embedding:

$$
h: \underline{H}_{6} \times[0,1] \rightarrow \underline{\underline{H}} \times[0,1] .
$$

1st step. Using Edward's version of the torus furling methods of [EK], as explained in Proposition 4.9 of [S], we get canonically from $h$ a homeomorphism $\bar{h}$ of $B^{k} \times T^{n} \times Q \times[0,1]$, which is the identity if $h$ is, and has the following properties:

(*) $\bar{h} \mid S^{k-1} \times T^{n} \times Q \times[0,1]$ is the identity.

$\bar{h}=h$ on $B^{k} \times 5 B^{n} \times Q \times[0,1]$, when we identify $B^{k} \times 5 B^{n} \times Q \times$ $[0,1]$ with its image by the quotient map

$$
q: B^{k} \times \mathbf{R}^{n} \times Q \times[0,1] \rightarrow B^{k} \times\left(\mathbf{R}^{n} / 8 Z^{n}\right) \times Q \times[0,1]=B^{k} \times T^{n} \times Q \times[0,1] .
$$

Now $\bar{h}$ lifts canonically, up the covering map $q$, to a homeomorphism $g_{1}$ of $B^{k} \times \mathbf{R}^{n} \times Q \times[0,1]=\underline{\underline{H}} \times[0,1]$, which has the properties:

(*) $g_{1} \stackrel{\partial H}{=} \times[0,1]$ is the identity,

(i) $g_{1}$ is the identity if $h$ is,

(ii) $g_{1}=\bar{h}=h$ on $\underline{H}_{5} \times[0,1]$.

By standard covering space theory, $g_{1}$ commutes with all translation of the covering, so that $g_{1}$ and $g_{1}^{-1}$ are uniformly continuous; and even more: the rule $h \mapsto g_{1}$ is continuous if the source space is equipped with the compact open topology and the target (i.e. the space of uniformly continuous homeomorphisms of $\underline{\underline{H}} \times[0,1]$ with uniformly continuous inverse) has the uniform topology (which makes it into a topological group). From now on in this section, unless otherwise specified, our constructions will be canonical relative to these topologies.

2nd step (which contains the only really new idea). We need the following results whose proof is sketched in $\S 4$ :

Proposition 1.4. Let I be $[0,1]$. There exists a homeomorphism $\theta$ : $\underline{\underline{H}} \times I^{2} \rightarrow \underline{\underline{H}} \times I$ such that:

(i) $\bar{d}(\theta(x), p(x))$ tends to zero as $x$ tends to infinity (here $p$ denotes the projection $\underline{\underline{H}} \times I \times I \rightarrow \underline{\underline{H}} \times I$ which forgets the $2^{d}$ interval).

(ii) $\overline{\bar{\theta}} \underline{\underline{H}} \times 0 \times 0) \stackrel{=}{=} \times 0$,

(iii) $\theta$ commutes with the projection onto the factor $B^{k}$ of $\underline{\underline{H}}$.

Using 1.4 we define uniformly continuous homeomorphism $g_{2}$, which depends canonically on $g_{1}$ for the uniform topology, as follows: crossing $g_{1}$ with the identity of the second interval $I$, we obtain a homeomorphism $g_{1} \times \mathrm{id}_{I}$ of $\underline{\underline{H}} \times I^{2} ;$ put $g_{2}=\theta \circ\left(g_{1} \times \mathrm{id}_{I}\right) \circ \theta^{-1}$. 


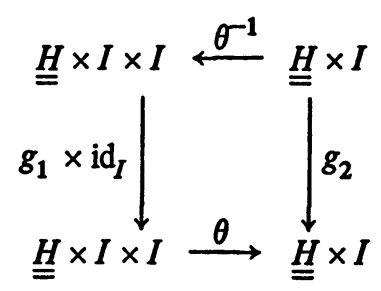

$g_{2}$ has the following properties:

(*) $g_{2} \mid \partial H \times[0,1]$ is the identity,

(i) $d\left(g_{1}(x), g_{2}(x)\right)$ tends to zero as $x$ tends to $\infty$ in $\underline{\underline{H}} \times[0,1]$,

(ii) $\left.\left.g_{2} \stackrel{\underline{H}}{=} \times\{0\}\right) \subset \theta \underline{\underline{H}} \times[0,1] \times 0\right)$ which is a fixed $Z$-set of $\underline{\underline{H}} \times[0,1]$, independent of $g_{2}$.

3rd step. By Chapman's canonical unknotting of $Z$-sets in $Q$-manifolds (see Theorem 5.1 and the apparatus of $\left[C_{1}\right]$ ), we can canonically arrange that $g_{2}$ becomes the identity on $\underline{H}_{5} \times\{0\}$, without changing it on $\underline{\underline{H}} \times[0,1]$ nor outside some compact set. Indeed the fact that $\left.g_{2} \underset{\underline{H}}{H_{5}} \times 0\right)$ lies in a fixed compact Z-set (independent of $g_{2}$ ) is enough to be able to apply Chapman's theorem; furthermore, it is not explicitly stated in $\left[\mathbf{C}_{\mathbf{1}}\right]$ that the canonical unknotting can be made with compact support, but it is an immediate consequence of the statement given there.

So we have now a canonical rule $g_{2} \mapsto g_{3}$ such that:

(*) $g_{3} l \partial H \times[0,1]$ is the identity.

(i) $g_{3}=g_{2}$ outside some compact set (independent of $g_{2}$ ), and therefore $d\left(g_{3}(x), g_{1}(x)\right)$ tends to 0 as $x$ tends to $\infty$.

(ii) $g_{3} \underline{H}_{5} \times 0$ is the identity.

Condition (i) implies, in particular, that the rule $g_{2} \mapsto g_{3}$ is continuous for the uniform topology.

4th step. Let $J$ be a radial homeomorphism of $\mathbf{R}^{n}$ onto $5 B^{n}$, which is the identity on $4 B^{n}$. Crossing with the identity of $B^{k} \times Q \times[0,1]$, it leads to a "compression" homeomorphism of $\underline{\underline{H}} \times[0,1]$ onto $\underline{\underline{H}}_{5} \times[0,1]$, that we still denote by $J$.

Property (i) of $g_{3}$ implies, together with the uniform continuity of $g_{1}^{-1}$, that $d\left(g_{1}^{-1} \circ g_{3}(x), x\right)$ tends to 0 as $x$ tends to $\infty$; hence the following map $g_{4}$ : $\underline{H} \times[0,1] \longrightarrow \underline{\underline{H}} \times[0,1]$ is a well-defined homeomorphism:

$$
g_{4}(x)=\left\{\begin{array}{l}
x \quad \text { outside } \underline{\underline{H}}_{5} \times[0,1], \\
J \circ\left(g_{1}^{-1} \circ g_{3}\right) \circ J^{-1}(x) \text { for } x \text { in } \underline{\underline{H}}_{5} \times[0,1] .
\end{array}\right.
$$

Note that:

(*) $g_{4} \mid \underline{\underline{\partial H}} \times[0,1]$ is the identity, 
$g_{4}=g_{1}^{-1} \circ g_{3}=h^{-1}$ on $\underline{H}_{3} \times 0$, by properties (ii) of $g_{1}$ and $g_{3}$ (if all the maps are close to the identity),

The rule $g_{1} \mapsto g_{4}$ is canonical for the uniform topology, this uses strongly the fact that $g_{1} \mapsto g_{3}$ was so.

If we now restrict $g_{4}$ to $\underline{\underline{H}}_{6} \times[0,1], h_{1}=h g_{4}$ is an open embedding of $\underline{\underline{H}}_{6} \times[0,1]$ into $\underline{\underline{H}} \times[0,1]$, which equals $h$ outside $\underline{H}_{5} \times[0,1]$ and has the following properties:

(*) $h_{1} \underline{\partial H}_{6} \times[0,1]$ is the identity,

(**) $h_{1} \underline{\underline{H}}_{3} \times 0$ is the identity.

This ends the proof of the Weak Handle Lemma.

2. Proof of the Deformation Principle (see precise statement in §0). As it is explicitly underlined in [S], such a Deformation Principle holds once we prove it for a basis of open sets, namely the relatively compact open sets whose closure is contained in a chart (in fact a $Q$-manifold can always be covered by two charts, but this yields no simplification here). Therefore by standard compactness we need only to prove $D^{\prime}(\underline{\underline{n}} ; \underline{\underline{D}}, \underline{\underline{V}}, \underline{\underline{C}} ; \underline{\underline{U}})$, where $U$ is an open subset of $I^{n}, C$ a compact subset of $U, D$ and $V$ are closed subsets of $I^{n}$ with $V$ a neighborhood of $D$.

Proof of $D^{\prime}(\underline{\underline{I}} ; \underline{\underline{D}}, \underline{\underline{V}}, \underline{\underline{C}} ; \underline{\underline{U}})$. We consider two cases, according as $C$ meets the boundary $\partial U$ of the (finite-dimensional) manifold $U$, or not.

1st case. $C \cap \partial U=\varnothing$.

This case follows from 1.3, by taking a small handle decomposition of $I^{n}$. The proof follows exactly the lines of [EK]; see it for details.

2nd case. $C \cap \partial U \neq \varnothing$.

Take a collared neighborhood of $\partial U$ in $U$. We identify this neighborhood with $\partial U \times[0,1]$; through this identification $\partial U$ is identified with $\partial U \times 1$. We can always suppose (by restricting the collar near $\partial U \times 1$ and reparametrizing by $[0,1])$ that there exist compact neighborhoods $N_{1}$ and $N_{2}$ of $\overline{C-V} \cap(\partial U \times$ $0)$ in $\partial U \times 0$ such that:
(i) $N_{1} \subset \stackrel{\circ}{N}_{2}$,
(ii) $\left(C-\stackrel{\circ}{V} \cap(\partial U \times[0,1]) \subset \stackrel{\circ}{N}_{1} \times[0,1]\right.$,
(iii) $N_{2} \times[0,1] \cap D=\varnothing$.

By the first case applied to the closed set $\left[D \cup\left(C \cap\left(\partial U-\stackrel{\circ}{N}_{1}\right) \times[0,1]\right)\right]$ $\times Q$ and the compact set $\left.[(C-\partial U \times] 0,1]) \cup N_{2} \times 0\right] \times Q$, we can suppose that we are dealing with open embeddings which are the identity on these sets. Now using a small handle decomposition of $N_{2}$ and applying Chapman's Handle Lemma 1.1, it is easily seen (as in the 1st case) that we can straighten the open embeddings on the remaining part of $C \times Q$ which is included in $N_{1} \times$ $[0,1] \times Q$. This finishes the proof of the Deformation Principle. 
REMARK. The proof is almost the same as in the finite-dimensional case, but we proceed in "reverse order": "we straighten first the interior and then the boundary". We are obliged to do so because if we cross a finite-dimensional manifold $M$ with $Q$, then $\partial M \times Q$ is no longer a topological invariant.

3. Some corollaries. We give here a proof of the following result (see [AK, p. 59]):

THEOREM 3.1. There does not exist any topological group structure on a Q-manifold.

Proof. Suppose that $M$ is a $Q$-manifold supporting a topological group structure; take any neighborhood $V$ of the neutral element of $M$, whose compact closure lies in a chart $U$. By restricting $V$ we can suppose that $V \cdot V \subset U$. Transporting the whole situation into the Hilbert cube, we have a point $x_{0}$ in $Q$, neighborhoods $V$ and $U$ of $x_{0}$, with $\bar{V} \subset U$, and a continuous rule $x \mapsto h_{x}$ associating to each point in $V$ an open embedding $h_{x}: V \rightarrow U$ with the property $h_{x}\left(x_{0}\right)=x$ (use the translation by $x$ ). Now, by application of the Deformation Principle to the open set $V$ and the compactum $C=\left\{x_{0}\right\}$, we obtain canonically for $x$ near $x_{0}$ an open embedding $h_{x}^{\prime}: V \rightarrow U$ such that:

(i) $h_{x}^{\prime}\left(x_{0}\right)=x_{0}$,

(ii) $h_{x}^{\prime}=h_{x}$ outside some compact set independent of $x$.

To each $x$ near $x_{0}$, associate the homeomorphism of $Q$ defined by:

$$
\left\{\begin{array}{l}
g_{x}=h_{x} \circ h_{x}^{\prime-1} \text { on } h_{x}^{\prime}(V), \\
g_{x}=\text { id outside } h_{x}^{\prime}(V) .
\end{array}\right.
$$

Note that $g_{x}\left(x_{0}\right)=x$, so we have obtained a local cross section of the evaluation map at $x_{0}, p: H(Q) \rightarrow Q$ from the homeomorphism group of $Q$ onto $Q$ ( $p$ is defined by $p(h)=h\left(x_{0}\right)$ ); thus we have shown that $p: H(Q) \rightarrow Q$ is a locally trivial principal bundle (with structural group the space of homeomorphisms of $Q$ fixing $\left.x_{0}\right)$. The base $Q$ being contractible, the bundle is trivial; so there exists a global section $s: Q \rightarrow H(Q)$ (i.e. $s(x)\left(x_{0}\right)=x$ for any $x$ in $Q$ ). Choose any point $x_{1} \neq x_{0}$ and define $f: Q \rightarrow Q$ by $f(x)=s(x)\left(x_{1}\right)$. It is easily seen that $f$ has no fixed point, but this contradicts the fact that $Q$ (being a compact AR) has the fixed point property (see [B, p. 101]).

We list now some corollaries of the Deformation Theorem. The proofs are completely formal once given the Deformation Theorem, so we do not repeat these proofs but merely give exact references to the places where they can be found.

THEOREM 3.2. If $M$ is a compact $Q$-manifold, then the homeomorphism group $H(M)$ of $M$ is locally contractible. 
Proof. Apply the Deformation Theorem with: $D=V=\varnothing$ and $C=U$ $=M$. $\square$

Remark. As explained to us by T. A. Chapman this theorem can also be deduced from 1.1.

THEOREM 3.3. If $h_{t}: U \rightarrow M, t \in I$, is an isotopy of open embeddings in the $Q$-manifold $M$, then, given any compact set $C \subset U$, one can extend $h_{t} \mid C$ to an ambient isotopy of $M$.

Proof. See [EK, p. 79] or [S, p. 147]. 口

THEOREM 3.4. A proper submersion whose fibers are $Q$-manifolds is a locally trivial bundle map.

Proof. See [CK, p. 151] or [S, p. 150-152]. 口

THEOREM 3.5. There is only a countable number of compact Q-manifolds, up to homeomorphism.

Proof. See [CK, p. 149] or [S, p. 159-162].

THEOREM 3.6. Let $G$ be a finite group, $M$ a compact $Q$-manifold and $F A(G, M)$ be the space of free $G$-actions on $M$ endowed with the compact open topology. Then $\mathrm{FA}(G, M)$ is locally contractible. Moreover two free $G$-actions on $M$ isotopic through G-actions are equivalent.

Proof. See [E, §2]. 口

4. Appendix. We sketch here the construction of the homeomorphism $\theta$ used in the 2nd step of the proof of the Weak Handle Lemma. such that:

PROPOSITION 4.1. There exists a homeomorphism $\theta: \underline{\underline{H}} \times I^{2} \rightarrow \underline{\underline{H}} \times I$

(i) $d(\theta(x), p(x))$ tends to zero as $x$ tends to infinity in $\underline{H} \times I^{2}$ (here $p$ denotes the projection $\underline{\underline{H}} \times I^{2} \rightarrow \underline{\underline{H}} \times I$ forgetting the second interval).

(ii) $\theta \underline{\underline{H}} \times 0 \times 0)=\underline{\underline{H}} \times 0$.

(iii) $\theta$ commutes with the projection onto the factor $B^{k}$ of $\underline{\underline{H}}$.

The existence of $\theta$ will follow from:

Proposition 4.2. There exists a pseudo isotopy $h_{u}: Q \times I^{2} \rightarrow Q \times I$ (defined for $0 \leqslant u \leqslant 1$ ) such that:

(i) $h_{u}$ is a homeomorphism for $u<1$.

(ii) $h_{1}$ is the projection $Q \times I^{2} \rightarrow Q \times I$ forgetting the second interval.

(iii) $h_{u}(q, 0,0)=(q, 0)$ for every $q$ in $Q$. 
Proof that $4.2 \Rightarrow 4.1$. Let $\varphi$ be a function $B^{k} \times \mathbf{R}^{n} \rightarrow[0,1$ [ obtained by composition of the projection $B^{k} \times \mathbf{R}^{n} \rightarrow \mathbf{R}^{n}$, and a function $\mathbf{R}^{n} \rightarrow[0,1$ [ which tends to 1 at infinity. Define $\theta:\left(B^{k} \times \mathbf{R}^{n}\right) \times\left(Q \times I^{2}\right) \rightarrow\left(B^{k} \times \mathbf{R}^{n}\right) \times$ $(Q \times I)$ by $\theta(x, y)=\left(x, h_{\varphi(x)}(y)\right)$ where $x \in B^{k} \times \mathbf{R}^{n}$ and $y \in Q \times I^{2}$. Trivially, $\theta$ verifies the conditions of 4.1.

Now since the pair $(Q \times I, Q \times 0)$ is homeomorphic to $(Q \times Q, Q \times 0)$, it is clear that 4.2 follows (by crossing everything with $Q$ ) from:

Proposition 4.3. There exists a pseudo isotopy $\bar{h}_{u}: Q \times I \rightarrow Q$ such that:

(i) $\bar{h}_{u}$ is a homeomorphism for $u<1$,

(ii) $\bar{h}_{1}$ is the projection on $Q$,

(iii) $\bar{h}_{u}(0,0)=0$ for every $u \in[0,1]$.

Proof of 4.3. Using some standard coordinates permutation techniques, 4.3 follows easily from the fact the homeomorphism $g:[0,1]^{3} \rightarrow[0,1]^{3}$, defined by $g\left(t_{1}, t_{2}, t_{3}\right)=\left(t_{2}, t_{3}, t_{1}\right)$ is isotopic to the identity by an isotopy which leaves $(0,0,0)$ fixed. $\square$

\section{REFERENCES}

[AK] R. D. Anderson and N. Kroonenberg, Open problems in infinite-dimensional topology, Louisiana State Univ., 1973 (preprint).

[B] K. Borsuk, Theory of retracts, PWN, Warsaw, 1967. MR 35 \#7306.

[C ${ }_{1}$ ] T. A. Chapman, Canonical extensions of homeomorphisms, General Topology and Appl. 2 (1972), 227-247. MR 46 \#9924.

$\left[\mathrm{C}_{2}\right] \longrightarrow$, Homeomorphisms of Hilbert cube manifolds, Trans. Amer. Math. Soc. 182 (1973), 227-239. MR 51 \#9067.

[ $\left.\mathrm{C}_{3}\right]$, Notes on Hilbert cube manifolds, Univ. of Kentucky, 1973 (preprint).

$\left[\mathrm{C}_{4}\right] \longrightarrow$, Concordance of Hilbert cube manifolds, Trans. Amer. Math. Soc. 219 (1976), 253-268.

[CK] J. Cheeger and J. Kister, Counting topological manifolds, Topology 9 (1970), 149-151. MR 41 \#1055.

[EK] R. D. Edwards and R. C, Kirby, Deformations of spaces of imbeddings, Ann. of Math. (2) 93 (1971), 63-88. MR 44 \#1032.

[S] L. C. Siebenmann, Deformation of homeomorphisms on stratified sets, Comm. Math. Helv. 47 (1972), 123-163.

[E] A. Edmonds, Local connectivity of spaces of group actions, Quart. J. Math. Oxford Ser. (2) 27 (1976), 71-84.

MATHÉMATIQUE BATIMENT 425, UNIVERSITÉ DE PARIS-SUD, CENTRE D'ORSAY, 91405 ORSAY, FRANCE 